\title{
BrainSuite: An Automated Cortical Surface Identification Tool
}

\author{
David W. Shattuck and Richard M. Leahy \\ Signal and Image Processing Institute, Department of Electrical Engineering, \\ University of Southern California, Los Angeles, California USA 90089-2564 \\ \{shattuck, leahy\}@sipi.usc.edu
}

\begin{abstract}
We describe a new magnetic resonance (MR) image analysis tool that produces cortical surface representations with spherical topology from MR images of the human brain. The tool provides a sequence of low-level operations in a single package that can produce accurate brain segmentations in clinical time. The tools include skull and scalp removal, image nonuniformity compensation, voxel-based tissue classification, topological correction, rendering, and editing functions. The collection of tools is designed to require minimal user interaction to produce cortical representations. In this paper we describe the theory of each stage of the cortical surface identification process. We then present validation results using real and phantom data.
\end{abstract}

\section{Introduction}

Surface representations of the human cerebral cortex are important for visualization and analysis of neuroimaging data [1] and as constraints for localizing functional activation from magneto-encephalography (MEG) and electroencephalography (EEG) data [2,3]. MR imaging offers neuroanatomical detail, but extraction of cortical surfaces from MR imagery faces several problems, including measurement noise, partial volume effects, and image nonuniformity due to magnetic field inhomogeneities. In this paper we describe a new tool that provides a comprehensive approach to extracting a representation of the cerebral cortex from T1-weighted MR images.

The problems we address in our work have been addressed by many others. Wells et al. presented an Expectation-Maximization (E-M) approach to image nonuniformity and tissue classification [4]. Kapur et al. combined the E-M approach with morphological and active contour methods to isolate and segment the brain [5], and later incorporated Gibbs and geometric priors into the E-M method to improve the classifier [6]. Sled et al. addressed the problem of nonuniformity using an E-M approach that estimates a gain field to sharpen the histogram of the MR image; this field is kept smooth using a cubic B-spline [7]. Software for this method is publicly available via the Internet (http://www.bic.mni.mcgill.ca/software/N3/) under the name Non-parametric Non-uniform intensity Normalization, or N3. 
The general problem of identifying the cortex in MR imagery has been approached from several directions. Atlas-based approaches to segmentation do not typically perform well in the cortex due to intersubject variability. To overcome this problem, Collins et al. combined their atlas-based method (ANIMAL) with a low-level method (INSECT) [8]. Active contour methods often perform best after initialization with a low-level segmentation, as described by Xu et al. and Pham et al. $[9,10]$. They find the cerebral cortex using fuzzy C-means classification. The fuzzy membership set is then repeatedly median-filtered until a topologically spherical isocontour is found; this contour represents the boundary between white matter and grey matter. A gradient vector flow method is then used to move this boundary to the medial layer of the cortex. Zeng et al. presented an identification sequence that uses a level-set method to find coupled surfaces representing the interior and exterior boundary of the cerebral cortex [11]. Dale et al. described a complete method for cortical surface identification that first smoothes image nonuniformities with a cubic spline that normalizes white matter peaks throughout the image, then strips skull and scalp using a deformable template, and finally labels the white matter based on intensity and neighborhood information [12]. Hand-editing is required to correct topological defects in the segmentation prior to tessellation. The tessellated surface is then deformed to refine the cortex.

The tool we describe in this paper is based on a sequence of low-level operations, resulting in a fast yet accurate method to identify the cortex. First, skull and scalp are removed from the image using edge detection and morphological processing $[13,14]$. The stripped brain is then processed to remove image nonuniformities using a parametric model that adjusts local intensities of the image to match global properties [14]. Next, the intensity corrected brain is classified at the voxel level into key tissue types: white matter, grey matter, cerebrospinal fluid, and combinations of these [14]. The white matter corresponding to the telencephalon is selected and further processed to ensure that key neuroanatomical structures, such as the ventricles, are interior to the initial white matter volume. This volume is then processed using a graph-based approach to remove topological inconsistencies in the volume, which is the most novel aspect of our approach [15]. The final volume may then be tessellated using the Marching Cubes algorithm [16] to produce a cortical surface that is topologically equivalent to a sphere.

\section{Methods}

\subsection{Removal of Extraneous Tissue}

Removal of non-brain tissue from the MR volume facilitates later stages in the algorithm as fewer voxels and fewer tissue types are involved. We refine the brain extraction procedure initially described in [13] to remove skull, scalp, and other tissue from the MR image. This portion of the algorithm has been publicly released as the Brain Surface Extractor (http://neuroimage.usc.edu/bse/), and is currently in use in several neuroanatomical studies. The process begins with an 
anisotropic diffusion filter [17], which smoothes contiguous tissue regions while respecting the edge boundaries that occur between these regions. We then use a three-dimensional Marr Hildreth edge detector [18], which produces a binary image with edges that are closed boundaries. These edges separate the volume into several distinct objects. We seek the object whose boundary represents the space between the brain and the skull and dura; this object is assumed to be the largest central connected component in the binary edge image.

The use of the anisotropic diffusion filter enhances this boundary, but some connections between the brain and other tissues will still remain due to noise and small anatomical structures such as the optic nerves. Simply finding the largest central connected component in the edge volume will leave several other structures attached to the brain. We use morphological erosion with a $3 \mathrm{D}$ rhombus operator of radius one to break these attachments from the brain prior to labeling the connected components. The desired component is then dilated to restore it to approximately its original size. A morphological closing operation with an octagonal element of size four is applied to fill surface pits that may be present due to small errors in the edge detection operation. This operation will also smooth some aspects of the surface detail. However, this will be recovered in later operations when the voxels are classified according to tissue type.

\subsection{Image Nonuniformity Compensation}

Inhomogeneneity in the magnetic fields during image acquisition and magnetic susceptibility variations in the scanned subjects cause intensity nonuniformities in MR images that prevent characterization of voxel tissue content based solely on image intensity. As a result, segmentation as well as quantitative studies of MR images require compensation for these nonuniformities. We use a parametric tissue measurement model to estimate the local variations in the gain field of the image by comparing the intensity properties of the whole MR volume with the properties of local neighborhoods within the image.

We extend the tissue measurement model of Santago and Gage [19], which describes the probability of measuring a particular intensity given the relative fractions of key tissue types including partial volume types. We incorporate a spatially variant bias term, $b_{k}$, which describes the nonuniformity effect at the $k$-th voxel measurement, $x_{k}$ :

$$
p\left(x_{k} \mid b_{k}\right)=\sum_{\gamma \in \Gamma} p(\gamma) p\left(x_{k} \mid \gamma, b_{k}\right),
$$

where $\gamma$ is the tissue class from the set $\Gamma$ including white matter (WM), grey matter (GM), cerebrospinal fluid (CSF), and partial volume combinations of these (WM/GM, GM/CSF, and CSF/Other). The function $p(x \mid \gamma, b)$ is determined by the tissue types present in the particular voxel. For voxels composed of a single tissue type (pure voxels),

$$
p(x \mid \gamma, b)=G_{b \mu_{\gamma}, \sigma}(x),
$$


where $G_{\mu, \sigma}(x)$ is a Gaussian density function of mean $\mu$ and variance $\sigma^{2}$. The pure tissue voxels have nonstationary mean values that vary multiplicatively from a global mean value, $\mu_{\gamma}$, according to the bias $b_{k}$. For mixed tissue types,

$$
p(x \mid \gamma, b)=\int_{0}^{1} G_{\alpha b \mu_{a}+(1-\alpha) b \mu_{b}, \sigma}(x) d \alpha,
$$

where $\alpha$ is a mixture parameter assumed to be a uniformly distributed random variable as per Santago and Gage, $\mu_{a}$ and $\mu_{b}$ are the global mean values of the pure tissue types associated with the mixture class $\gamma$. For each particular image, we compute a priori estimates of the global tissue mean values and noise variance from automated analysis of the stripped brain's intensity histogram.

We assume the bias in the image changes very slowly spatially. Within a neighborhood centered about a particular voxel, we approximate the bias as constant. The mixture model (1) then describes the measurements made within the entire region, conditioned on knowing the fractional tissue content and bias of the region.

We select a uniformly spaced lattice of points in the image at which we estimate the bias field. The measurements taken in a neighborhood about each point are described by the mixture model allowing us to fit our model to a normalized histogram of the region. This provides estimates of both the tissue fractions and the bias within the region. We next use a set of outlier rejection steps on the bias estimates to eliminate poor fits between the model and the histogram. Because the bias field is smooth and slowly varying it is sufficient to estimate its values at a coarser sampling than the original image. We then use a tri-cubic B-spline to smooth and interpolate the robust estimate points, providing us with an estimated value for the bias field at each point in the image. We finally remove the bias from the image by dividing the intensity value of each voxel by its corresponding bias estimate.

\subsection{Partial Volume Classification}

Compensation for the nonuniformity in the MR image greatly simplifies the tissue classification problem. However, noise is still present in the system. For the most part, the brain image can be described by regions of contiguous tissue type. This allows us to use a Gibbs' prior that incorporates a spatial model for the brain tissues into our classification scheme. The model we have selected is quite simple:

$$
p(\Lambda)=\frac{1}{Z} \exp \left[-\beta \sum_{k} \sum_{j \in N_{k}} \delta\left(\lambda_{k}, \lambda_{j}\right)\right],
$$

where $\Lambda=\left\{\lambda_{1}, \lambda_{2}, \ldots \lambda_{|\Lambda|}\right\}$ is the set of labels describing the image, $Z$ is a scaling constant to ensure that we have a proper density function and $\beta$ controls the degree of influence of the prior on the voxel labels. $N_{k}$ is the D18 neighborhood (neighbors share an edge or face) about the $k$-th voxel. The $\delta$ terms govern the 
likelihood of different tissue labels being neighbors, hence we set $\delta\left(\gamma_{k}, \gamma_{j}\right)$ to -2 if labels $k$ and $j$ are identical, -1 if they have a common tissue type, and 1 if they have no common tissues. These scores are scaled according to the inverse of the distance of voxel $k$ to voxel $j$. In this way, the model penalizes configurations of voxels that are not likely to occur in the brain, e.g., white matter directly adjacent to CSF, while encouraging more likely types, e.g., white matter next to a partial volume mixture of white and grey matter.

We assume that our nonuniformity correction method has performed its task, and remove the bias terms in our image measurement model. We then use Bayes' formula to create a Maximum A Posteriori (MAP) classifier, which maximizes

$$
p(\Lambda \mid X)=\frac{p(X \mid \Lambda) p(\Lambda)}{p(X)},
$$

where $p(X \mid \Lambda)=\prod_{k} p\left(x_{k} \mid \lambda_{k}\right)$, with $p(x \mid \lambda)$ as in equation (1), but with the bias assumed uniform, i.e., $p(x \mid \gamma)=p(x \mid \gamma, b=1)$. Equation (5) is maximized using an iterated conditional modes (ICM) algorithm after initialization with a maximum likelihood classification.

\subsection{Constraining the Topology of the Cortical Surface}

The cerebral cortex is a single sheet of connected tissue that encloses the telencephalon. This topology begins during the early development of the embryo, and remains during normal development of the cerebrum. We assume the cortical surface to be topologically equivalent to a sphere after closing the cortex at the brainstem. Errors in the tissue classification stage and the limited resolution of the MR image acquisition as compared to the details of the neuroanatomy will result in cortical surface representations that do not have the appropriate topology.

BrainSuite includes the Topological Constraint Algorithm (TCA) presented in [15]. TCA is an iterative correction procedure that decomposes a volumetric object into a graph representation from which topological equivalence to a sphere may be determined. Slices along a particular axis of the volume are examined in turn. First, the foreground connected components in each slice are labeled. Then, connectivity between each of these components and the components in adjacent slices are determined. These connections are used to form a graph, where each connected component is a vertex in the graph and each connection between components is represented as a weighted edge between the corresponding vertices. The weight of each edge represents the strength of connection between two components. The graph captures both topological and geometric information about the object. The process is repeated for the background voxels, creating a second graph. It is our conjecture that if these graphs are trees then the object being analyzed is homeomorphic to a sphere.

TCA performs this graph analysis and then determines a pair of desired trees using a maximal spanning tree algorithm. In this way, the algorithm determines the edges corresponding to the minimal collection of voxels that need to be 
removed from the graphs to force them to be trees. These edges correspond to regions in the object where small topological handles or holes exist. The algorithm then edits these areas to remove the handles or holes. The process is applied iteratively along each axis, making the smallest changes possible with each iteration. This ensures that the smallest total change to the volume is made. The result of this procedure is an object that, when tessellated with the Marching Cubes algorithm, will be topologically equivalent to a sphere.
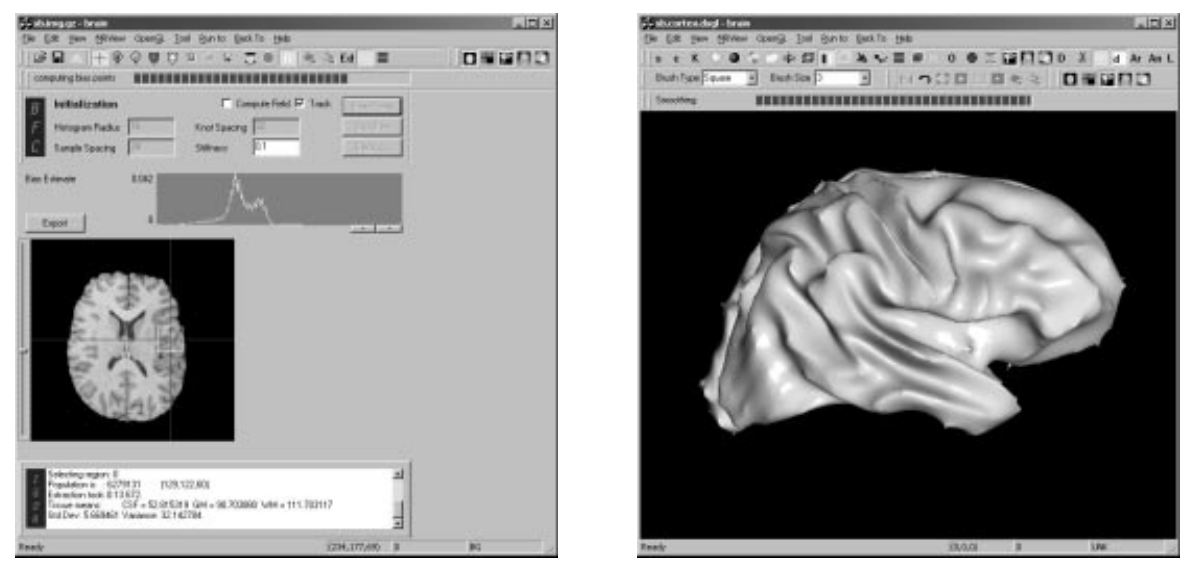

Fig. 1. The BrainSuite graphical user interface during (left) bias correction and (right) surface visualization of a highly smoothed cortical surface.

\section{Results and Discussion}

\subsection{Implementation}

We have implemented the sequence of operations into a single, stand-alone interactive tool using Microsoft Visual C++ (see Fig. 1) for use on the Microsoft Windows NT/2000 platforms. The program can be used on common desktop computer hardware to produce cortical volumes within minutes. BrainSuite guides the user through each stage of cortical surface identification and includes hand-editing and connected component tools to allow the user to perform segmentation correction if necessary. Each stage of processing may be performed independently, providing increased flexibility. In addition to the functionality described in this paper, BrainSuite also includes tools for visualization, smoothing, and inflation of cortical surfaces. BrainSuite has been developed to work with BrainStorm, the MEG/EEG Matlab toolbox produced by Baillet et al.[2], and provides the ability to visualize brain activation information on cortical surfaces. 


\subsection{Validation}

The Brain Surface Extractor (BSE) has been used extensively by numerous groups so we do not focus on its accuracy in this paper [20]. BSE was used as the first step for each of the volumes examined in this section. Thus, its performance will directly affect the results of each subsequent stage. In this section we present results for the nonuniformity correction, tissue classification, and topological constraint stages of the algorithm. Validation was performed on phantom and real data.

Nonuniformity Correction Assessing the performance of image nonuniformity correction in real data is problematic due to the lack of a ground truth. For this reason, we test our bias correction algorithm on the BrainWeb Phantom produced by the McConnell Brain Imaging Centre at the Montreal Neurological Institute [21]. The phantom provides a ground truth phantom image and simulated images of the phantom corrupted by noise and RF nonuniformity, available for download via the Internet (http://www.bic.mni.mcgill.ca/brainweb/). We tested our algorithm on the normal brain database with each available set of artifacts.

Ignoring quantization effects, intensity-threshold based classification methods are unaffected by changes of scale and translations of the image intensity. For this reason we compute a Procrustean metric that is the minimum root mean square difference between the two images being compared accounting for all possible translations and scalings of the corrected image intensity,

$$
e(\boldsymbol{y}, \tilde{\boldsymbol{x}})=\min _{a, b} \sqrt{\frac{1}{|\Omega|} \sum_{k \in \Omega}\left(y_{k}-\left(a \hat{x}_{k}+b\right)\right)^{2}},
$$

where $\hat{x}_{k}$ is the corrected image intensity of the $k$-th voxel, $\Omega$ is the region of interest, and $y_{k}$ is the intensity of the $k$-th voxel of the phantom. This metric provides a fair comparison of performance between different bias methods.

\begin{tabular}{|c|ccc|ccc|ccc|}
\hline RF Field: & \multicolumn{3}{|c|}{$0 \%$} & \multicolumn{3}{c|}{$20 \%$} & \multicolumn{3}{c|}{$40 \%$} \\
Bias Correction: & None & N3 & BFC & None & N3 & BFC & None & N3 & BFC \\
\hline $3 \%$ Noise & 2.98 & 3.02 & 3.13 & 4.13 & 3.83 & 2.97 & 6.23 & 4.30 & 3.51 \\
$5 \%$ Noise & 4.88 & 4.93 & 4.98 & 5.50 & 5.77 & 4.81 & 7.05 & 5.88 & 4.87 \\
$7 \%$ Noise & 6.65 & 6.81 & 6.75 & 6.96 & 7.24 & 6.55 & 8.05 & 7.33 & 6.58 \\
$9 \%$ Noise & 8.30 & 8.60 & 8.39 & 8.41 & 8.82 & 8.16 & 9.15 & 8.92 & 8.09 \\
\hline
\end{tabular}

Table 1. Normalized root mean square difference computed between ground truth phantom image and scaled noisy biased phantoms, before and after correction by BFC and N3. Values shown are as a percentage of the ground truth WM intensity. RF field values describe the strength of the simulated nonuniformity field. 
To examine the bias correction results specifically, a single mask generated by BSE was used for each image. The stripped brains were then processed by BFC using a single set of parameters for all volumes, and by N3 using the default parameters. We then compared the corrected images to the ground truth using (6) divided by the intensity of WM in the ground truth image. These results are tabulated in Table 1. In the cases where noise was applied with no bias, the normalized RMS difference metric shows that N3 and BFC both left the phantom volumes relatively unchanged. N3 performed slightly better on the $3 \%$ and $5 \%$ noise phantoms, while BFC performed slightly better on the $7 \%$ and $9 \%$ cases. In every case with simulated bias fields, the BFC-corrected image was closer to the original than the corresponding N3-corrected image. In most cases the RMS difference was very near to that of the unbiased image with the same level of noise, signifying that we have removed most of the variation attributable to inhomogeneity effects.
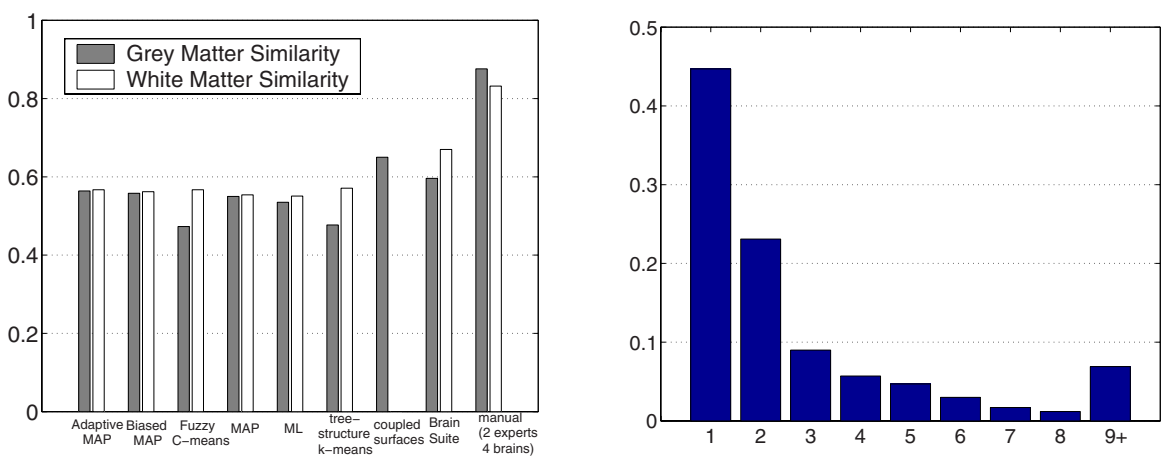

Fig. 2. (left) Similarity comparison computed for several methods using the IBSR dataset. (right) Average fraction of total changes made during each iteration of topological correction. Iteration number represents the largest number of voxels allowed to change to fix a topological problem.

Tissue Classification We tested our tissue segmentation method on data from the Internet Brain Segmentation Repository. The 20 normal MR brain data sets and their manual segmentations were provided by the Center for Morphometric Analysis (CMA) at Massachusetts General Hospital and are available at http://neuro-www.mgh.harvard.edu/cma/ibsr. The data provided by CMA were selected because they have been used in published studies and have various levels of difficulty. A few of these volumes have low contrast and relatively large intensity gradients, and the performance of the tested algorithms is poor on these. The volumes have slice dimensions of $256 \times 256$, with resolution of $1 \mathrm{~mm} \times 1 \mathrm{~mm}$. Interslice distance is $3 \mathrm{~mm}$, with the number of slices for each volume between 60 and 65 . 
BrainSuite was used to segment each of the brains in a minimally interactive fashion. We performed skull stripping using one of three parameter settings. Bias correction was performed using the same settings for all twenty brains. The selection of the tissue prior weighting for tissue classification was performed manually, using one of three settings. Settings were only varied when the results were clearly unacceptable. No manual editing of the brain volume or labels was performed. It is possible that more tuning of the parameters to the individual data would produce improved results.

To analyze our performance we use the Jaccard similarity metric, which measures the similarity of two sets as the ratio of the size of their intersection divided by the size of their union. CMA provides reference results for several methods that have been tested using these data; the methods are described in [22]. The results of each method are averaged over the twenty volumes. These averages, our own results, and the grey matter measure provided in [11] are shown in Fig. 2 (white matter metrics are not provided in [11]). Also shown are reference metrics for interoperator variability, 0.876 for GM and 0.832 for WM, proposed by CMA based on an interoperator variability comparison of two experts segmenting four brains. The best average performance of the six reference methods is 0.564 for GM and 0.571 for WM. Our method produced average similarity measures of 0.595 for GM and 0.664 for WM. This is significant since our method seeks the GM/WM boundary. The GM similarity measure for the coupled surface result on the whole brain is 0.657 , which outperforms our method [11].

It should also be noted that some of the volumes had artifacts that were beyond the capability of our program to generate useful cortical surfaces. Two of the volumes produced results poor enough to prevent further identification of the cortex. Our methods should achieve better performance on more recently acquired data, with voxel dimensions that are less anisotropic.

Topological Constraint Algorithm We processed 18 of the 20 IBSR brain volumes using the BrainSuite tools to produce inner-cerebral masks. The brainstem and cerebellum were removed manually. Some subcortical structures such as the ventricles and subcortical grey matter are not always well-segmented and were filled using a user-guided flood-filling. This process required approximately three to five minutes per brain. These white matter masks were then processed using the topological constraint algorithm. In each case, TCA successfully produced an object with spherical surface topology, as verified by computing the Euler characteristic on a Marching Cubes tessellation of the object.

The actual changes made to each volume were very minimal, with at most a $0.7 \%$ change in membership to the white matter set. This level of change is well within the variability one would expect to see among segmentations by experts. Figure 2 shows the average percentage of changes made with each iteration. The iteration number corresponds to the largest number of voxels changed to fix a specific topological problem. Figure 2 emphasizes that most changes are made in the very early stages of the algorithm and typically correspond to changes 

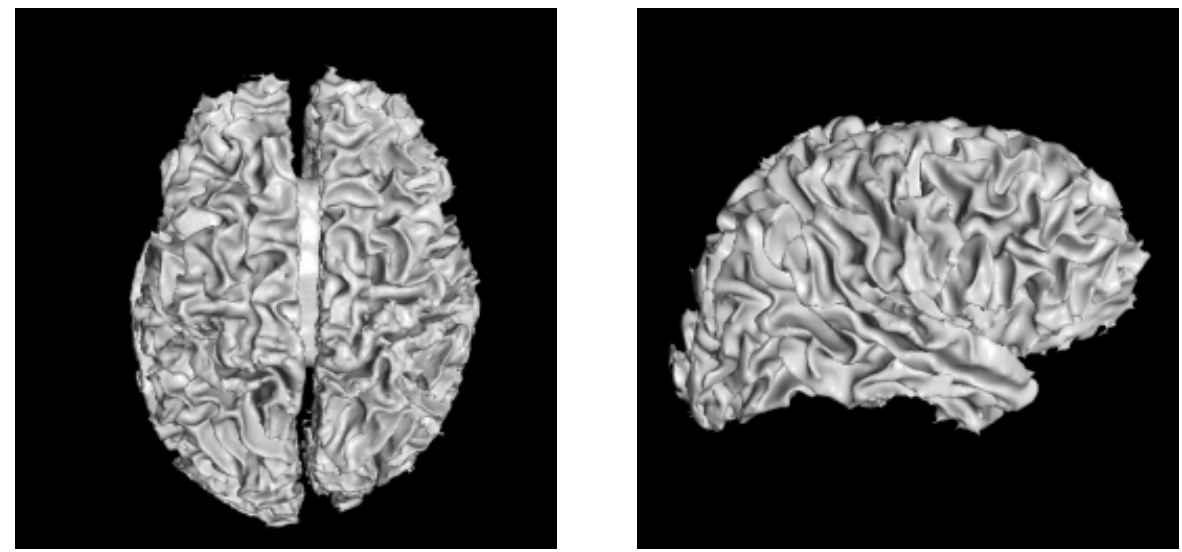

Fig. 3. Axial and sagittal views of a cortical surface with spherical topology, generated using BrainSuite. The surface represents the boundary between white matter and cortical grey matter.

of only a few voxels at a specific location. On newer, higher resolution data our method achieved much better results, and $75 \%$ of the topological corrections were achieved in the first iteration [15]. Figure 3 shows renderings of a topologically spherical cortical surface obtained from a high-resolution T1-weighted MR volume using BrainSuite. In this case, less than $0.1 \%$ of the initial set membership was changed by the topological constraint algorithm.

Processing Time Table 2 shows the CPU time of each stage of the algorithm applied to the IBSR data. Processing was performed on a $933 \mathrm{MHz}$ Intel Pentium III Xeon with $256 \mathrm{~K}$ cache and $256 \mathrm{MB}$ of RAM. The topological constraint algorithm is the newest component in the toolset, and may be optimized in future work to reduce the total time required for segmentation. High resolution data requires more processing time; the cortical volume in Figure 3 was generated in approximately fifteen minutes. Presently, cortical volumes may be identified from MR images in less than 30 minutes of total operator time using BrainSuite.

\begin{tabular}{|c|rrrrr|}
\hline & BSE & BFC & PVC TCA Total \\
\hline average & 5.1 & 51.8 & 3.4 & $4: 01$ & $5: 01$ \\
best case & 3.8 & 38.7 & 0.3 & $2: 18$ & $3: 06$ \\
worst case & 8.0 & $1: 11.8$ & 7.8 & $5: 40$ & $6: 33$ \\
\hline
\end{tabular}

Table 2. CPU times (in minutes:seconds) for each stage of the algorithm applied to the 20 normal MR brain data sets from the IBSR. 


\subsection{Discussion}

We have presented a self-contained toolset that produces topologically spherical cortical surface representations from T1-weighted MR images using an integrated sequence of low-level processing. The processing requires minimal user interaction, and our method was shown to work in very reasonable time on modern desktop computer hardware. The results of our cortical identification technique were validated on both phantom and real data, and were shown to outperform several existing methods.

\section{Acknowledgements}

This work was supported in part by the National Institute of Mental Health Grant RO1-MH53213.

\section{References}

1. Thompson, P.M., MacDonald, D., Mega, M.S., Holmes, C.J., Evans, A.C., Toga, A.W.: Detection and mapping of abnormal brain structure with a probabilistic atlas of cortical surfaces. Journal of Computer Assisted Tomography 21 (1998) $567-581$

2. Baillet, S., Mosher, J., Leahy, R., Shattuck, D.: Brainstorm: A Matlab toolbox for the processing of MEG and EEG signals. NeuroImage 9 (1999) S246

3. Dale, A.M., Sereno, M.I.: Improved localization of cortical activity by combining EEG and MEG with MRI cortical surface reconstruction : a linear approach. Journal of Cognitive Neuroscience 5 (1993) 162-176

4. Wells, W.M., Grimson, W.E.L., Kikinis, R., Jolesz, F.A.: Adaptive segmentation of MRI data. IEEE Transactions on Medical Imaging 15 (1996) 429-442

5. Kapur, T., Grimson, W.E.L., Wells, W.M., Kikinis, R.: Segmentation of brain tissue from magnetic resonance images. Medical Image Analysis 1 (1996) 109-127

6. Kapur, T., Grimson, W.E.L., Kikinis, R., Wells, W.M.: Enhanced spatial priors for segmentation of magnetic resonance imagery. In Wells, W.M., Colchester, A.C.F., Delp, S., eds.: Medical Image Computing and Computer-Assisted InterventionMICCAI '98. Lecture Notes in Computer Science, Vol. 1496. Springer-Verlag, Berlin Heidelberg (1998) 457-468

7. Sled, J.G., Zijdenbos, A.P., Evans, A.C.: A nonparametric method for automatic correction of intensity nonuniformity in MRI data. IEEE Transactions on Medical Imaging 17 (1998) 87-97

8. Collins, D.L., Zijdenbos, A.P., Baaré, W.F., Evans, A.C.: ANIMAL+INSECT : Improved cortical structure segmentation. In Kuba, A., Sámal, M., Todd-Pokropek, A., eds.: Information Processing in Medical Imaging: Proceedings of the 16th International Conference. Lecture Notes in Computer Science, Vol. 1613. SpringerVerlag, Berlin Heidelberg (1999) 210-223

9. Xu, C., Pham, D.L., Rettmann, M.E., Yu, D.N., Prince, J.: Reconstruction of the central layer of the human cerebral cortex from MR images. IEEE Transactions on Medical Imaging 18 (1999) 467-480

10. Pham, D., Prince, J.: Adaptive fuzzy segmentation of magnetic resonance images. IEEE Transactions on Medical Imaging 18 (1999) 737-752 
11. Zeng, X., Staib, L., Schultz, R.T., Duncan, J.: Segmentation and measurement of the cortex from 3-D MR images using coupled-surface propagation. IEEE Transactions on Medical Imaging 18 (1999) 927-937

12. Dale, A.M., Fischl, B., Sereno, M.I.: Cortical surface-based analysis I: Segmentation and surface reconstruction. NeuroImage 9 (1999) 179-194

13. Sandor, S., Leahy, R.: Surface-based labeling of cortical anatomy using a deformable database. IEEE Transactions on Medical Imaging 16 (1997) 41-54

14. Shattuck, D.W., Sandor-Leahy, S.R., Schaper, K.A., Rottenberg, D., Leahy, R.: Magnetic resonance image tissue classification using a partial volume model. Signal and Image Processing Institute Technical Report, University of Southern California 334 (2000)

15. Shattuck, D.W., Leahy, R.M.: Topologically constrained cortical surfaces from MRI. In Hanson, K.M., ed.: Medical Imaging 2000: Image Processing. Proceedings of the SPIE, Vol. 3979. (2000) in press

16. Lorensen, W., Harvey, E.: Marching cubes: A high resolution 3D surface construction algorithm. ACM Computer Graphics 21 (1987) 163-169

17. Gerig, G., Kubler, O., Kikinis, R., Jolesz, F.: Nonlinear anisotropic filtering of MRI data. IEEE Transactions on Medical Imaging 11 (1992) 221-232

18. Marr, D., Hildreth, E.: Theory of edge detection. Proceedings of Royal Society of London 207 (1980) 187-217

19. Santago, P., Gage, H.D.: Quantification of MR brain images by mixture density and partial volume modeling. IEEE Transactions on Medical Imaging 12 (1993) 566-574

20. Rehm, K., Shattuck, D., Leahy, R., Schaper, K., Rottenberg, D.: Semi-automated stripping of T1 MRI volumes: consensus of intensity- and edge-based methods. NeuroImage 9 (1999) S86

21. Collins, D., Zijdenbos, A., Kollokian, V., Sled, J., Kabani, N., Holmes, C., Evans, A.: Design and construction of a realistic digital brain phantom. IEEE Transactions on Medical Imaging 17 (1998) 463-468

22. Rajapakse, J.C., Kruggel, F.: Segmentation of MR images with intensity inhomogeneities. Image Vision Computing 16 (1998) 165-180 